Special issue of the 3rd International Conference on Computational and Experimental Science and Engineering (ICCESEN 2016)

\title{
Position Control of a Turret Using LabVIEW
}

\author{
A. SAYGIN ${ }^{a, *}$ AND A.M. RASHID ${ }^{b}$ \\ ${ }^{a}$ Gazi University, Faculty of Technology Department Electrical and Electronics Engineering, Ankara, Turkey \\ ${ }^{b}$ Gazi University, Graduate School of Natural and Applied Science, Ankara, Turkey
}

\begin{abstract}
Servo motors are commonly used for axial motion control of gun turrets. In this study, horizontal and vertical position control around two axes of motion of a gun turret was realized using LabVIEW software. Communication with Siemens s7-300 CPU315-2 PN/DP PLC has been realized using KEPServerEX software and using OPC tools in LabVIEW. KEPServerEX software was used to transfer motor control and status data between the PC and PLC. PLC was used to control the AC servo drivers using ProfiBUS protocol, in accordance with commands sent from LabVIEW. Servo motors with connected S110 CU305DP servo drivers have been controlled according to control data received from the PLC. Scout software was used for uploading communication and position commands and for changing factory defaults of AC servo drivers. Furthermore, status control and input of commands have been realized using a touch panel. All PLC and touch panel programming have been performed on TIAPortal V13SP1. Thus, the position control of gun turret has been provided using both, PC and the touch panel.
\end{abstract}

DOI: 10.12693/APhysPolA.132.970

PACS/topics: 06.60.Sx, 07.07.Tw

\section{Introduction}

Servo motors are commonly used in systems requiring a critical position control. AC servo motors can be controlled using motor drivers. Servo motor drivers obtain commands from PLCs. JOG, HOME, ABSOLUTE or RELATIVE movement commands can be sent to AC servo motors using a defined communication protocol between PLC and servo driver. Encoder position data of the AC servo motor can be read by servo driver and send to PLC. The desired position data can be also sent from PLC to servo driver. PID controller parameters of servo driver control motor movement according to the load.

PLCs can control servo motor movement using loaded program, whereas the desired position data can be also send from PC or any microprocessor.

If there is a free port protocol in PLC, the control commands can be supplied according to received data. If communication is set between the PC and PLC, OPC (object linking and embedding - OLE for process control) protocol has to be used. PLC producing companies use supervisory control and data acquisition - SCADA software for remote controlling. These SCADA software use there own OPC code and do not allow sharing there possibilities with the external software. If communication is set independently between PLC and PC, KEPServerEX software has to be used. Matlab or LabVIEW can be used for the desired position calculation. Communication between PLC and KEPServerEX can be set up using OPC tools of packaged software.

Zuluaga B.J.G. et al. have emphasized in their study that MAX485 chip is not safe for external communication, for ProfiBUS communication, between PLC and

\footnotetext{
*corresponding author; e-mail: asaygin@gazi.edu.tr
}

an embedded system [1]. Ling Zhou, Jie Chen and Lixin $\mathrm{Xu}$ have realized remote control of asynchronous motor in a study of Siemens PLC and Freeport communication application [2]. Laurentiu Schiop and Marian Gaiceanu have realized software control of blending tank, using Siemens PLC and man to machine communication interface [3]. Bai Van and Zhang Hongwei have succeeded at controlling of entire automatic washing machine with S7-200 PLC [4]. Communication between PC and PLC was realized using Freeport and the designed Visual Basic interface [5]. Tri Oktaviana Putri et al. have ensured temperature control of a pasteurizer system. Only PLC and a touch panel have been used for communication [6].

Different protocols are preferred for different industrial applications. Freeport protocol is generally used for communication between PC and PLC. Although actual Freeport communication data rate is between $9600 \mathrm{Kbps}$ and $1920 \mathrm{Kbps}$ in literature, this rate is not enough for a complicated application.

PLC were also used in various applications for control of systems with different communication methods [7-10]. Dindar at al. have designed within LabVIEW and implemented in NI-PXI platform a new robotic telescope [11], without using PLC. This application included universal motion interface device for control of servo systems.

In this study, Siemens S7-300 CPU315-2 PN/DP PLC was chosen for the project. S110 CU305DP PLC was used as the control unit for the AC servo. PLC and servo driver were connected using ProfiBUS communication protocol, for which the Ethernet protocol is preferred. In addition, KEPServerEX was used for OPC.

\section{Control scheme of servo driver}

Designed system consisted of three mechanical components. These are PC, PLC and servo systems. LabVIEW and KEPServerEX software were used in the PC. TIAPortal was used in PLC. In addition, Scout software was used in servo systems. 
The desired position data of the gun turret was calculated in LabVIEW. Data was transferred to PLC via KEPServerEX using the OPC tools. PLC was used to send the motor movement commands, which were coded with LADDER diagrams in TIAPortal, to the driver. PLC and the servo driver were connected through ProfiBUS. Servo driver has provided motor positioning movement according to commands received from the PLC. The actual position of servos was sent back to PLC. Furthermore, PLC has transfered this data, status data and the error data to PC.

Communication between servo motor and servo driver was realized with DriveCLIQ.

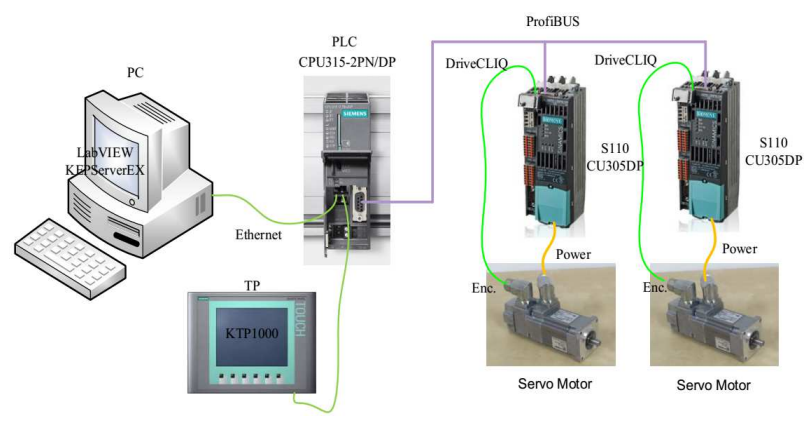

Fig. 1. System architecture.

Servo driver ensures servo motor movement using JOG, HOME, ABSOLUTE and RELATIVE commands. For the described application, two servo motors and two drivers were used, with every servo system controlling one axis of movement. System architecture is shown in Fig. 1.

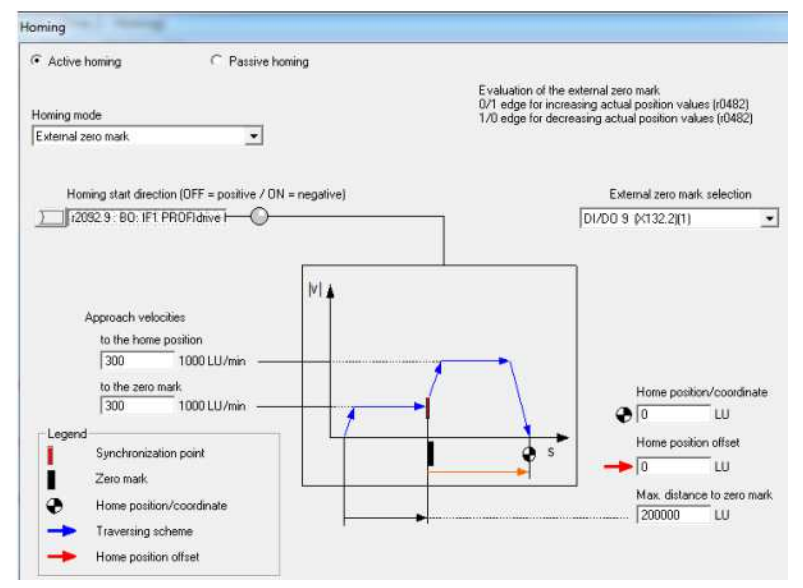

Fig. 2. Setup page of home reference signal of servo motor.

Signal input was realized for home process from the DI9 address using X132.2, due to using digital addresses of servo driver CU305DP. Network settings and external settings page was uploaded to the driver after editing in Scout software. The setup page of home reference signal of servo motor in Scout software is shown in Fig. 2.

\section{KEPServerEX}

KEPServerEX allows easier project development using one server interface. This interface is independent of the system type. Thus, it allows to communicate with more devices. This software enables easier communication centralizing and realizing for users. KEPServerEX provides single configuration method and program development tools for the users.

One of these tools is OPC server, providing unique performance and compatibility between different devices. Because of Windows-based study, it provides data transmitting to client application from several industrial devices and systems for 32 bit or 64 bit server applications.

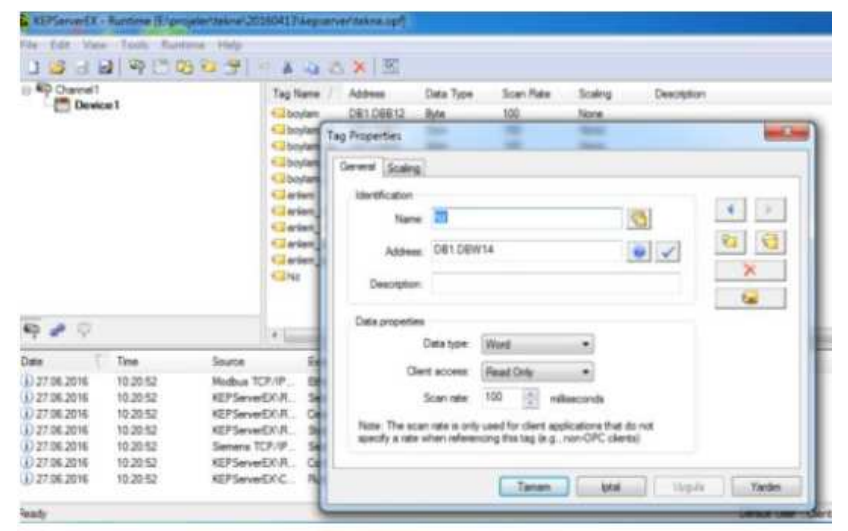

Fig. 3. Variables defined in KEPServerEX OPC server.

OPC type was selected for KEPServerEX [12]. Devices were added to the selected communication protocol. Variables providing reading and writing were set for each device. Types of variables are important. Because of using of marked or unmarked addresses one must be careful. The defined variables must be selected from the addresses assigned to PLC's global variables. Then communication was set up between the PC and PLC. Using DB addresses makes it easier with Siemens S7-300 PLC. Variables defined in KEPServerEX OPC server are shown in Fig. 3.

\section{LabVIEW project}

More than one PLC can connect with each other, using different methods, due to LabVIEW software. This software supplies real time data transferring between OLE control devices and man-to-machine interface for process control. A LabVIEW program consists of a block diagram and a front panel for user interface. Thanks to the front panel, data can be entered into the system and results can be presented, in an application designed in LabVIEW [13]. However, the block diagram is the part where the main work is done. On one hand controls are 
added to the front panel, on the other hand, virtual instruments are used in the block diagram. LabVIEW I/O server tools are shown in Fig. 4.

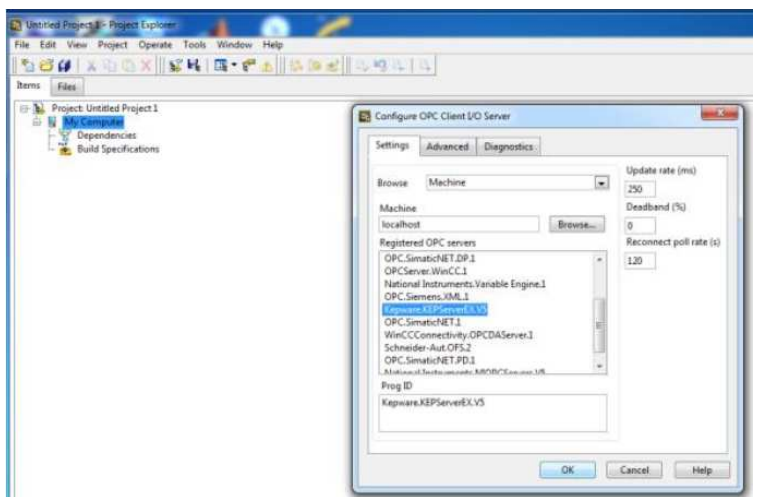

Fig. 4. LabVIEW I/O server tools.

\section{Experimental results}

During experimental studies, movement of servo motor to different positions was tested. New position and velocity data were sent from LabVIEW to PLC and the actual position data were received. Processes of movement to Home position or relative position changing process are explained in figures. For absolute position movement, motor movement is started after referencing is made.

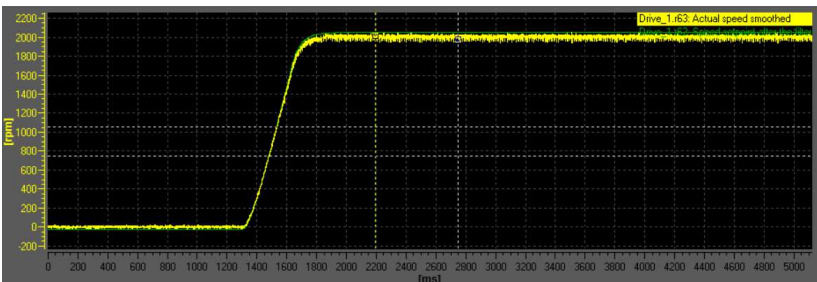

Fig. 5. Servo motor reaching $2000 \mathrm{rpm}$.

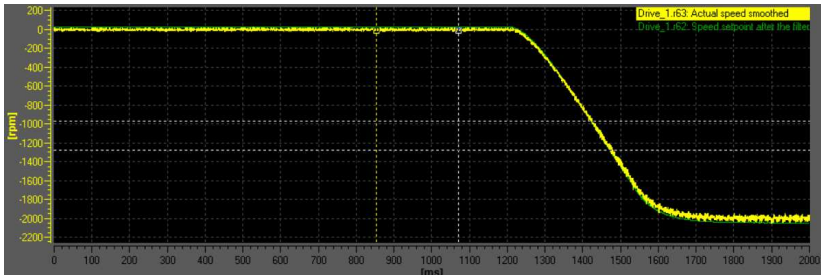

Fig. 6. Servo motor reaching $-2000 \mathrm{rpm}$.

Experimental curves of servo motor reaching $2000 \mathrm{rpm}$ and $-2000 \mathrm{rpm}$ are given in Figs. 5 and 6, respectively.

The desired and the actual velocity change of servo motor at $2000 \mathrm{rpm}$ and at $3000 \mathrm{rpm}$ are given in Figs. 7 and 8 , respectively and phase current changing of servo motor, while reaching rated speed is given in Fig. 9. Green line represents the reference velocity and the yellow line is the actual velocity in these three figures. In addition, that orange line is the changing current of motor, given in Fig. 9.

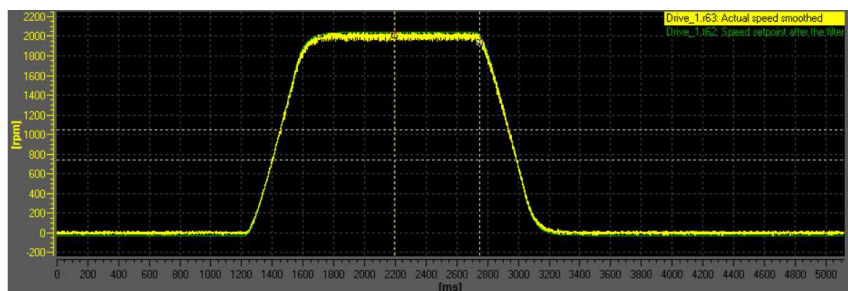

Fig. 7. The desired and the actual velocity change of servo motor at $2000 \mathrm{rpm}$.

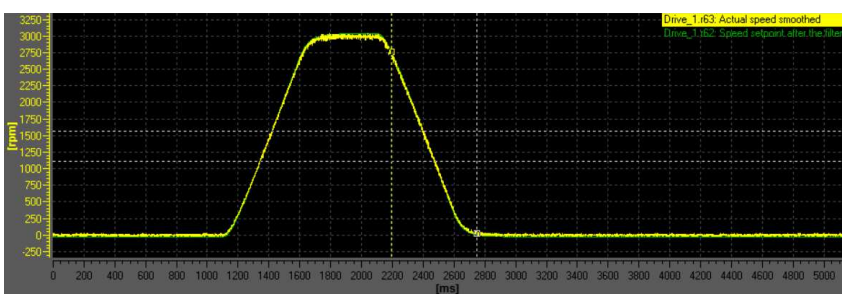

Fig. 8. The desired and the actual velocity change of servo motor at $3000 \mathrm{rpm}$.

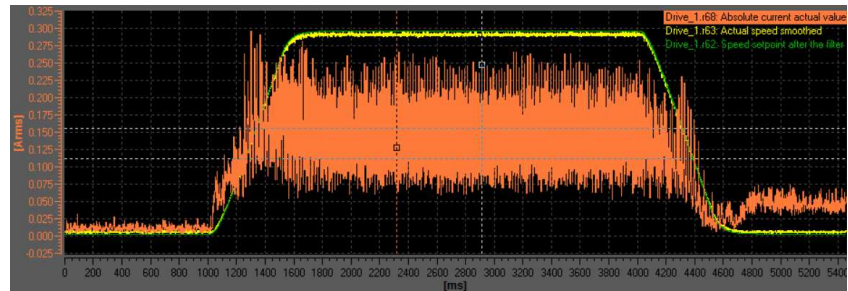

Fig. 9. Phase current changing of servo motor while reaching the rated speed.

\section{Conclusions}

In this study bidirectional data transfer between Siemens PLC and PC was realized using KEPServerEX OPC. Communication synchronization between PLC and $\mathrm{PC}$ was continuously provided in real-time.

Biaxial position and velocity control of servo motors was realized using LabVIEW. The desired position and velocity are controlled by variables in LabVIEW, values of which are transferred to CPU315-2 PN/DP using KEPServerEX OPC. The actual position is transferred back from PLC to LabVIEW and then the desired and the actual positions are validated in LabVIEW. The proper operation of biaxial gun turret application and the position control have been demonstrated.

\section{Acknowledgments}

We are grateful for trial versions of LabVIEW and KEPServerEX.

\section{References}

[1] Z.B.J. Gonzalo, H.C.J. Aurelio, S.G.S. Ignacio, in: 2014 XIX Symp. Image, Signal Processing and Artificial Vision, 2014, p. 1.

[2] L. Zhou, J. Chen, L. Xu, in: 2008 Int. Sem. Future BioMedical Information Engineering, 2008, p. 318.

[3] L. Schiop, M. Gaiceanu, in: 2010 3rd Int. Symp. Electrical and Electronics Engineering (ISEEE), 2010, p. 165. 
[4] B. Yan, Z. Hongwei, in: 2012 World Automation Congress (WAC), 2012, p. 1.

[5] H. Fang, W. Tang, in: 2012 2nd Int. Conf. Consumer Electronics, Communications and Networks (CECNet), 2012, p. 2795.

[6] T.O. Putri, Rahmadwati, B. Siswojo, in: Electrical Power, Electronics, Communications, Controls and Informatics Seminar (EECCIS-2014), 2014, p. 99.

[7] O.M. Pişirir, O. Bingöl, Acta Phys. Pol. A 130, 36 (2016).

[8] M. Imal, Acta Phys. Pol. A 130, 245 (2016).
[9] A. Aksoz, A. Saygin, Int. J. Electrical, Electronics Data Commun. 3, 19 (2015).

[10] Y. Debbağ, E.N. Yilmaz, in: 2015 3rd Int. Istanbul Smart Grid Congress and Fair (ICSG), 2015, p. 1.

[11] M. Dindar, S. Dindar, E. Kandemir, C. Bayar, S. Helhel, T. Ozisik, Acta Phys. Pol. A 128, B-73 (2015).

[12] Kepserver, 2016, [online], available: www.kepware.com/products/kepserverex/.

[13] Labview, 2016, [online], available: www.ni.com/labview/. 